\title{
Von Visiten und Visitationen
}

\section{Werner Bauer}

Dr. med., ehem. Präsident des Schweizerischen Instituts für ärztliche Weiter- und Fortbildung SIWF

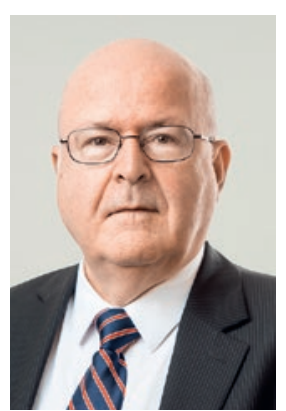

Zwei Begriffe, zwei Bestandteile des Spitallebens: Der eine beschreibt eine Aktivität, die sich im Laufe der Zeit beträchtlich verändert hat, der andere ein wichtiges Instrument zur Unterstützung der Facharztweiterbildung. Ich gehöre zur Generation, welche die Chefarztvisite noch als das wöchentliche Hauptereignis auf einer Spitalabteilung erlebt hat. Die Assistenzärztinnen und Assistenzärzte bereiteten sich manchmal bis in die Nacht hinein auf den zeremoniellen weissen Umzug von Bett zu Bett vor. Dieser formierte sich jeweils im Korridor und gliederte sich gemäss der Spitalhierarchie von den Oberärzten über die Stationsschwester bis zu den Unterassistentinnen und Unterassistenten. Ich erinnere mich an einen Chirurgen mit markantem Ego, bei dem der ganze Tross jeweils korrekt angetreten im Gang warten musste, bis der chefärztliche Ford Mustang in die Spitaleinfahrt brummte, der Chef die Ehrbezeugung der medizinischen Garde abnahm und zum ersten Patienten schritt. Auch an mein Herzklopfen erinnere ich mich, wenn der Internist mit skeptischem Blick nach dem Auskultieren meine roten und blauen Striche und Punkte in der Krankengeschichte kontrollierte, die das Atemgeräusch und die Herztöne zu beschreiben versuchten. Gelernt haben wir viel, aber die Steine, die nach einigermassen überstandener Visite immer einmal wieder von den Herzen der Betroffenen fielen, müssen in manchen Spitalböden Furchen hinterlassen haben.

Bei den Visiten hat sich inzwischen viel geändert. Mancherorts vielleicht sogar etwas gar viel? Das Pendel hat ausgeschlagen: einerseits in Richtung einer weniger autoritären Führung, andererseits aber auch in Richtung Beschleunigung der Abläufe, Zeitknappheit, Zunahme der Belastung von Ärztinnen und Ärzten jeder Stufe. Damit hat an vielen Orten die Weiterbildungstradition der Visite einen Teil ihres Stellenwerts verloren.

Und damit komme ich zu den Visitationen, einem entscheidenden Mittel zur Sicherung der Weiterbildungsqualität: Alle anerkannten Kliniken müssen nach einem Leiterwechsel visitiert werden. Es sind rund 150 pro Jahr - ein grosser, aber voll gerechtfertigter Aufwand für das Schweizerische Institut für ärztliche Weiterund Fortbildung (SIWF). Eine Weiterbildungsstätte wird von drei Expertinnen resp. Experten besucht. Eine Person vertritt das betreffende Fachgebiet, eine ist fachfremd und eine vertritt den Verband Schweizerischer
Assistenz- und Oberärztinnen und -ärzte (VSAO). Sie studieren vorbereitende Unterlagen und führen $\mathrm{Ge}$ spräche mit der Spitaldirektion, mit den Verantwortlichen der Weiterbildungsstätte, dem Kader und in absolut vertraulichem Rahmen mit den Assistenzärztinnen und -ärzten. Es ist bemerkenswert, wie schnell ein erfahrenes Visitationsteam das Lern- und Arbeitsklima einer Klinik erfassen, und wie offen mit den Assistenzärztinnen und -ärzten über die Sonnen- und Schattenseiten der Weiterbildung gesprochen werden kann.

Von Seiten der Spitaldirektion bekommt man mit schöner Regelmässigkeit zu hören, welch hoher Stellenwert der Weiterbildung beigemessen und wie aktiv sie unterstützt werde. Diese Schalmeien werden beim Gespräch mit den unteren Hierarchiestufen nicht selten etwas relativiert, bis man bei den Assistenzärztinnen und Assistenzärzten der Realität direkt ins Auge blickt. Im Durchschnitt ist diese Realität zwar befriedigend bis erfreulich und es kommt sogar vor, dass die Frage unbeantwortet bleibt, was sich die jungen Ärztinnen und Ärzte wünschen würden, wenn eine Fee durch den Raum wandelte. Leider gibt es aber doch Weiterbildungsstätten, die den Anforderungen nicht genügend gerecht werden können oder wollen. Die Einhaltung der Arbeitszeit und eine inakzeptable, als sinnlos empfundene administrative Belastung sind Themen, die immer wieder auftauchen. Daneben können die Visitationsteams auch auf klinikspezifische Schwachstellen wie einen mühsamen $\mathrm{Zu}$ gang zu den Kaderärztinnen und -ärzten, eine fehlende "unité de doctrine», undurchsichtige Dienstpläne oder eine überknappe Einführungszeit stossen.

Je nach Schweregrad eines Mangels wird er an der Schlussbesprechung diskutiert oder es werden im Visitationsbericht Empfehlungen oder verbindliche Auflagen formuliert. Die meisten Visitationen finden in einer kollegialen, fairen Atmosphäre statt und am Schluss bestätigen viele der Visitierten, sie hätten vor allem auch von der Vorbereitung und vom Gedankenaustausch profitiert. Es wäre erstaunlich, wenn nicht auch manchmal ein Team kühl, defensiv und abweisend empfangen würde oder wenn es nicht vereinzelte Ansätze zu «Potemkinschen Dörfern" gäbe, bei denen sich später erweist, dass da nicht ganz alles Gold war, was glänzte. Es gilt deshalb, einen wohlwollend-kritischen Blick zu haben.

Lehrreiche Visiten und konstruktive Visitationen: zwei wichtige Pfeiler der ärztlichen Weiterbildung. 